\title{
ON THE STRUCTURE OF DISSIPATIVE WAVES IN TWO AND THREE DIMENSIONS*
}

\author{
BY \\ LORNE HALABISKY** AND LAWRENCE SIROVICH \\ Brown University, Providence, Rhode Island
}

\begin{abstract}
The problem of two- and three-dimensional small disturbances in a dissipative gas is considered. Explicit forms in terms of known functions are obtained for the time-asymptotic theory in each case. Although the far field solutions may be nonlinear in one dimension, they are always self-consistently linear for two and three dimensions.

1. Introduction. In the present paper we consider the evolution of disturbances as governed by the linearized Navier-Stokes equations. A previous paper (Sirovich [8]) dealt with the one-dimensional case and in the present study the program is completed by the two- and three-dimensional results. This is therefore an appropriate time to sum up the results of the linearized theory and to also place it in perspective within the nonlinear framework.
\end{abstract}

In this connection we first comment that the main problem has been to find a fundamental solution. This solution plays two different roles in the theory. First, it may be used to construct the solution to an arbitrary initial value problem. This is briefly discussed in Secs. 2 and 3 below and more completely developed in [8]. A second use is in describing the solution to the linear problem for $t$ approaching $\infty$. The fundamental solution without further manipulations yields the solution for large times, at least when the proper significance is given to the constants that appear. Although the first application is the more important one, at least in the sense that it includes the second, we use the second to illustrate the results thus far obtained and its relation to the nonlinear theory.

To start this discussion we recall the results obtained by Sirovich [8] for one dimension. We denote the total mass, momentum and energy addition of the initial disturbance by $m, M$, and $E$ respectively. Then the period of final decay to lowest order is governed by

$$
\begin{aligned}
\nabla & \sim \frac{\exp \left(-x^{2} / 4 \nu t\right)}{(4 \pi \nu t)^{1 / 2}} \frac{\mathbf{q}_{0}^{1} \mathbf{q}_{0}^{1}}{\gamma} \cdot\left[\begin{array}{c}
m \\
M \\
E
\end{array}\right] \\
& +\frac{\exp \left(-(x-\sqrt{\gamma} t)^{2} / 4 \sigma t\right)}{(4 \pi \sigma t)^{1 / 2}} \frac{\mathbf{q}_{0}^{2} \mathbf{q}_{0}^{2}}{2 \gamma} \cdot\left[\begin{array}{c}
m \\
M \\
E
\end{array}\right]+\frac{\exp \left(-(x+\sqrt{\gamma} t)^{2} / 4 \sigma t\right)}{(4 \pi \sigma t)^{1 / 2}} \frac{\mathbf{q}_{0}^{3} \mathbf{q}_{0}^{3}}{2 \gamma} \cdot\left[\begin{array}{c}
m \\
M \\
E
\end{array}\right]
\end{aligned}
$$

* Received October 16, 1969. The results presented in this paper were obtained in the course of research sponsored by the Office of Naval Research under contract Nonr 562(39) with Brown University.

** Present address: University of British Columbia, Vancouver. 
where $\boldsymbol{\nabla}=[\rho, u, T]$ represents the perturbation density, velocity, and temperature respectively and $\mathbf{q}_{0}^{i} \mathbf{q}_{0}^{i}(i=1,2,3)$ are constant matrices defined in [8]. The normalization is discussed in Sec. 2. These results have been derived under the assumption that $t \gg 1$. For the large-time asymptotic case, in addition, $t$ is large compared to the time for a sound wave to traverse the initial disturbance (see [8] and Sec. 6 below).

The solution (1.1) is concentrated in three regions of maximal asymptotic behaviorthe wave regions moving to the right and left and the contact region which remains at the origin. In view of the fact that the regions are disengaged for large times, we may write, using an obvious notation,

$$
\nabla \sim \nabla_{+}+v_{0}+\nabla_{-} .
$$

The basic assumption characterizing the linearization of the nonlinear Navier-Stokes equations is that

$$
\mathbf{u} \cdot \nabla \mathbf{u} \leqq \nabla^{2} \mathbf{u} .
$$

(All quantities are dimensionless under a normalization which leaves the Reynolds number $O(1)$.) Because our solution decouples (see (1.2)), we need to look at condition (1.3) in each of the regions. In particular, for the case of the right travelling velocity perturbation in (1.1),

$$
u(x, t) \sim \frac{Q}{2 \sqrt{\gamma}} \frac{\exp \left(-(x-\sqrt{\gamma} t)^{2} / 4 \sigma t\right)}{(4 \pi \sigma t)^{1 / 2}},
$$

and condition (1.3) becomes

$$
Q \ll 1
$$

where

$$
Q=m+\sqrt{\gamma} M+\chi E .
$$

The following points should be noted. First, even though $u$ (1.4) is small in magnitude, it is not valid for all time unless (1.5) holds. Secondly, even if the perturbed initial data

$$
\nabla(x, t=0)=\nabla_{0}(x)
$$

is small, the linear theory is not valid for $t \rightarrow \infty$ unless the integral of the perturbed initial data satisfies (1.5). (In this case it is, however, correct on shorter time scales including the initial instant.) Alternatively, even though the initial data is large pointwise at $t=0$, the linear theory is valid for $t \rightarrow \infty$ if (1.5) holds.

For the case where the convective terms are of the same order as the dissipative terms, a nonlinear theory may be given which leads to Burger's equation. This is not surprising, however, in view of the work previously done by Su and Gardner [10], Moran and Shen [6], Lighthill [5], and Hayes [4]. These results constitute the subject of a forthcoming paper.

We next consider the two- and three-dimensional solutions. Here the solutions decouple into two regions of maximum asymptotic behavior-the wave regir,ns moving outward from the origin and the contact region which remains at the origin. The contact region consists of two parts, a vorticity region structured by viscosity and an entropy region structured by thermal conductivity.

Considering the wave region only, the two-dimensional perturbation velocity describ- 
ing the period of final decay is given by

$$
\begin{aligned}
& u(R, t) \sim \frac{Q}{\sqrt{\gamma}} \frac{\Gamma\left(\frac{3}{4}\right) M\left(\frac{3}{4}, \frac{1}{2},-\left(\left(R-(\sqrt{\gamma} t)^{2} / 4 \sigma t\right)\right)\right.}{8 R^{1 / 2} \pi^{3 / 2}(\sigma t)^{3 / 4}} \\
& +\frac{Q}{\sqrt{\gamma}} \frac{\Gamma\left(\frac{5}{4}\right)(R-\sqrt{\gamma} t) \exp \left(-(R-\sqrt{\gamma} t)^{2} / 4 \sigma t\right)}{8 R^{1 / 2} \pi^{3 / 2}(\sigma t)^{5 / 4}} M\left(\frac{1}{4}, \frac{3}{2},(R-\sqrt{\gamma} t)^{2} / 4 \sigma t\right) .
\end{aligned}
$$

For three dimensions we have

$$
u(r, t) \sim \frac{Q}{\sqrt{\gamma}} \frac{(r-\sqrt{\gamma} t)}{2 r(4 \pi \sigma t)^{3 / 2}} \exp \left(-(r-\sqrt{\gamma} t)^{2} / 4 \sigma t\right) .
$$

( $M(a, b, z)$ is the confluent hypergeometric function and $R$ and $r$ denote the two- and three-dimensional radii respectively.) These results are derived below in Secs. 4, 5 and 6 under the same assumptions as in the one-dimensional case. Applying (1.7) and (1.8) to our criteria for self-consistency of linearization (1.3), we find that the two-dimensional counterpart to (1.5) is

$$
Q / t^{3 / 4} \ll 1
$$

and for three dimensions

$$
Q / t^{3 / 2} \ll 1
$$

It follows that the flow field is always self-consistently linear for sufficiently large times in two and three dimensions, independently of the initial disturbance.

We mention in passing that when the time is such that (1.9) or (1.10) is violated, a nonlinear theory resembling that of Burger's equation has been derived and this shorter time regime may be analytically described. These results will be included in the aforementioned report of our work.

Finally we mention that certain aspects of the present study have been considered by Ryžhov [7]. Ryžhov examined the decay of perturbations in cylindrical and spherical sound impulses. In particular, he showed that the structure of waves and the asymptotic relationships of their decay when time approached infinity were related to the effects of viscosity and thermal conduction.

2. Formulation. We introduce the equilibrium quantities

$$
\rho_{0}, \quad T_{0}, \quad a_{0}^{2}=\left(\partial p_{0} / \partial \rho_{0}\right)_{T_{0}}, \quad c_{v}=\left(\partial e_{0} / \partial T_{0}\right)_{\rho_{0}},
$$

a yet unspecified length scale $L$ and the following normalized perturbed quantities [8]: $\tilde{\mathbf{x}}=\mathbf{x} / L, \quad \bar{t}=a_{0} t / L, \quad \bar{\rho}=\left(\rho-\rho_{0}\right) / \rho_{0}, \quad \tilde{\mathbf{u}}=\mathfrak{u} / a_{0}, \quad \tilde{T}=\left(c_{0} / a_{0}^{2} T_{0}\right)^{1 / 2}\left(T-T_{0}\right)$.

We drop all tildes with the understanding that all quantities are now dimensionless. The normalized, linearized Navier-Stokes equations are

$$
\frac{\partial}{\partial t}\left[\begin{array}{c}
\rho \\
\mathfrak{u} \\
T
\end{array}\right]+\left[\begin{array}{ccc}
0 & \nabla \cdot & 0 \\
\nabla-\delta \nabla \nabla \cdot-\eta \nabla^{2} & \chi \nabla \\
0 & \chi \nabla \cdot & -\xi \nabla^{2}
\end{array}\right]\left[\begin{array}{l}
\rho \\
\mathfrak{u} \\
T
\end{array}\right]=\left[\begin{array}{l}
0 \\
0 \\
0
\end{array}\right]
$$


where

$$
\eta=\mu /\left(\rho_{0} a_{0} L\right), \quad \delta=(\beta+\mu / 3) /\left(\rho_{0} a_{0} L\right), \quad \xi=\kappa /\left(\rho_{0} c_{0} a_{0} L\right), \quad \chi=(\gamma-1)^{1 / 2}
$$

and $\gamma=c_{p} / c_{0}=c_{0}^{2} / a_{0}^{2}$ with $c_{0}$ and $a_{0}$ being the adiabatic and isothermal speeds of sound respectively. Symbolically we write (2.1) as

$$
(\partial \nabla / \partial t)+\mathrm{A}(\nabla) \nabla=0 .
$$

We formally solve (2.3) by means of Fourier transforms. Define

$$
\nabla(\mathbf{k})=\int_{-\infty}^{\infty} \exp (+i \mathbf{k} \cdot \mathbf{x}) \mathbf{v}(\mathbf{x}) d \mathbf{x} .
$$

Notice that under the transformation $\nabla \rightarrow-i \mathbf{k}$, the transformed form of Eq. (2.3) is

$$
(\partial \nabla / \partial t)+\mathrm{A}(-i \mathbf{k}) \mathbf{v}=\mathbf{0} .
$$

It is convenient to introduce the fundamental matrix $\mathrm{V}^{n}(\mathrm{k}, t)(n=2,3)$ which satisfies

$$
\left(\partial \mathrm{V}^{n} / \partial t\right)+\mathrm{A}(-i \mathbf{k}) \mathrm{V}^{n}=0, \quad \mathrm{~V}^{n}(t=0)=1 .
$$

Then the solution of the transformed problem in an unbounded domain and for arbitrary initial data

$$
\nabla(t=0)=\nabla_{0}(\mathbf{x})
$$

is given simply by $\mathbf{\nabla}(\mathbf{k}, t)=\mathbf{V}^{\mathbf{n}}(\mathbf{k}, t) \nabla_{0}(\mathbf{k})$. Fourier inverting, we obtain

$$
\nabla(\mathrm{x}, t)=\mathrm{V}^{n}(\mathrm{x}, t) * \nabla_{0}(\mathrm{x})
$$

for the solution to (2.1) subject to the data (2.5). (The asterisk denotes space convolution.)

3. Nature of the asymptotic solution. It is now convenient to fix the length scale $L$ by choosing $\delta$ defined in (2.2) to be order unity. On physical grounds we know that this choice makes the remaining dissipative parameter $\xi$ and $\eta O(1)$. (From the point of view of kinetic theory the scale $L$ is proportional to the mean-free-path.) In this section we search for the time-asymptotic form of the fundamental matrix $\mathrm{V}^{n}(\mathbf{x}, t)$. Because of the choice of the length scale, the normalization time scale may be likened to the mean time between molecular collisions. Hence large times in the asymptotic sense will be very small on a macroscopic scale.

From (2.4) we have that

$$
\mathbf{V}^{n}(\mathbf{k}, t)=\exp [-\mathbf{A}(-i \mathbf{k}) t]
$$

and hence that

$$
\mathbf{V}^{\mathbf{n}}(\mathbf{x}, t)=\frac{1}{(2 \pi)^{n}} \int_{-\infty}^{\infty} \exp [-\imath \mathbf{k} \cdot \mathbf{x}-\mathbf{A}(-\imath \mathbf{k}) t] d \mathbf{k}
$$

where $n$ denotes the number of space dimensions. Also, from (2.6), the solution to an initial value problem in an unbounded domain is given by

$$
\mathbf{v}=\mathbf{V}^{n} * \nabla_{0}=\frac{1}{(2 \pi)^{n}} \int_{-\infty}^{\infty} \exp [-\imath \mathbf{k} \cdot \mathbf{x}-\mathbf{A}(-\imath \mathbf{k}) t] \mathbf{v}_{0}(\mathbf{k}) d \mathbf{k} .
$$


On denoting the matrix of eigenvalues of $\mathbf{A}(-i \mathrm{k})$ by $\mathrm{D}$ and the corresponding matrix of eigenvectors by $\mathrm{S}$, we may write

$$
A=\operatorname{SDS}^{-1}
$$

so that

$$
\mathbf{v}(\mathbf{x}, t)=\frac{1}{(2 \pi)^{n}} \int_{-\infty}^{\infty} \exp (-\imath \mathbf{k} \cdot \mathbf{x}) \mathbf{S}^{-\mathbf{D} t} \mathbf{S}^{-1} \cdot \nabla_{0} d \mathbf{k} .
$$

On representing the eigenvectors of $\mathrm{A}$ by $\mathrm{q}^{\mu}$ and the corresponding eigenvalues by $d^{*}$, the symmetry of A gives us the following simplified form:

$$
\mathbf{v}(\mathbf{z}, t)=\frac{1}{(2 \pi)^{n}} \sum_{\mu=1}^{n+2} \int_{-\infty}^{\infty} \exp \left[-\imath \mathbf{k} \cdot \mathbf{z}-d^{\mu}(\mathbf{k}) t\right] \frac{\mathbf{q}^{\mu} \mathbf{q}^{\mu}}{\mathbf{q}^{\mu} \cdot \mathbf{q}^{\mu}} \cdot \nabla_{0} d \mathbf{k}
$$

where as earlier $n$ represents the number of space dimensions.

The precise evaluation of the $d^{\mu}$ and $\mathbf{q}^{\mu}$ is extremely difficult; however, the perturbation expansion in small $\mathrm{k}$ is straightforward. The results for three dimensions are

$$
\begin{aligned}
& d^{1}=\lambda^{1}+O\left(k^{3}\right)=\nu k^{2}+O\left(k^{3}\right) \\
& \mathbf{q}^{1}=\mathbf{w}^{1}+O(k)=[\chi, 0,0,0,-1]+O(k) \\
& d^{2}=\lambda^{2}+O\left(k^{3}\right)=\eta k^{2}+O\left(k^{3}\right) \\
& \mathbf{q}^{2}=\mathbf{w}^{2}+O\left(k^{2}\right)=i\left[0, k_{2},-k_{1}, 0,0\right]+O\left(k^{2}\right) \\
& d^{3}=\lambda^{3}+O\left(k^{3}\right)=\eta k^{2}+O\left(k^{3}\right) \\
& \mathbf{q}^{3}=\mathbf{w}^{3}+O\left(k^{3}\right)=i\left[0,-k_{1} k_{3},-k_{2} k_{3}, k_{1}^{2}+k_{2}^{2}, 0\right]+O\left(k^{3}\right) \\
& d^{4}=\lambda^{4}+O\left(k^{3}\right)=i \sqrt{\gamma} k+\sigma k^{2}+O\left(k^{3}\right) \\
& \mathbf{q}^{4}=\mathbf{w}^{4}+O\left(k^{2}\right)=i\left[-k, k_{1} \sqrt{\gamma}, k_{2} \sqrt{\gamma}, k_{3} \sqrt{\gamma},-k \chi\right]+O\left(k^{2}\right) \\
& d^{5}=\lambda^{5}+O\left(k^{3}\right)=-i \sqrt{\gamma} k+\sigma k^{2}+O\left(k^{3}\right) \\
& \mathbf{q}^{3}=\mathbf{w}^{5}+O\left(k^{2}\right)=i\left[k, k_{1} \sqrt{\gamma}, k_{2} \sqrt{\gamma}, k_{3} \sqrt{\gamma}, k \chi\right]+O\left(k^{2}\right)
\end{aligned}
$$

where

$$
\sigma=\frac{1}{2}\left(\eta+1+\left(\chi^{2} \xi / \gamma\right)\right), \quad \nu=\xi / \gamma .
$$

The results for two dimensions are

$$
\begin{aligned}
& d^{1}=\lambda^{1}+O\left(k^{3}\right)=\nu k^{2}+O\left(k^{3}\right) \\
& \mathbf{q}^{1}=\mathbf{w}^{1}+O(k)=[\chi, 0,0,-1]+O(k) \\
& d^{2}=\lambda^{2}+O\left(k^{3}\right)=\eta k^{2}+O\left(k^{3}\right) \\
& \mathbf{q}^{2}=\mathbf{w}^{2}+O\left(k^{2}\right)=i\left[0, k_{2},-k_{1}, 0\right]+O\left(k^{2}\right) \\
& d^{3}=\lambda^{3}+O\left(k^{3}\right)=i \sqrt{ } \gamma k+\sigma k^{2}+O\left(k^{3}\right) \\
& \mathbf{q}^{3}=\mathbf{w}^{3}+O\left(k^{2}\right)=i\left[-k, k_{1} \sqrt{ } \gamma, k_{2} \sqrt{ } \gamma,-k \chi\right]+O\left(k^{2}\right) \\
& d^{4}=\lambda^{4}+O\left(k^{3}\right)=-i \sqrt{ } \gamma k+\sigma k^{2}+O\left(k^{3}\right) \\
& \mathbf{q}^{4}=\mathbf{w}^{4}+O\left(k^{2}\right)=i\left[k, k_{1} \sqrt{ } \gamma, k_{2} \sqrt{ } \gamma, k \chi\right]+O\left(k^{2}\right) .
\end{aligned}
$$


It should be noted that all coefficients above are $O(1)$ because of choice of our length scale $L$.

Following arguments by Sirovich [8] one may show that all $d$ 's have nonnegative real parts and $d=0$ if and only if $k=0$. Then from a general discussion of integrals of the type (3.3), under these conditions, the following approximation has been proven valid [9]:

$$
\begin{aligned}
\mathrm{V}^{n} & =\sum_{\mu=1}^{n+2} \frac{1}{(2 \pi)^{n}} \int_{-\infty}^{\infty} \exp \left(-i \mathbf{k} \cdot \mathbf{x}-\lambda^{\mu} t\right) \frac{\mathbf{w}^{\mu} \mathbf{w}^{\mu}}{\mathbf{w}^{\mu} \cdot \mathbf{w}^{\mu}} d \mathbf{k}+O^{*}\left(t^{-3 n / 4}\right) \\
& =\mathrm{V}_{\text {N. s. }}^{n}+O^{*}\left(t^{-3 n / 4}\right) .
\end{aligned}
$$

The symbol $O^{*}\left(t^{-p}\right)$ signifies a quantity of $O\left(t^{\delta-p}\right)$ where $\delta>0$ is arbitrarily small. (Again $n$ signifies the number of space dimensions.)

The solution to an initial value problem is given by

$$
\nabla(\mathbf{z}, t) \sim \mathrm{V}_{\mathrm{N} . \mathrm{s} .}^{n} * \mathrm{v}_{\mathbf{0}} .
$$

Again $\nabla_{0}$ is arbitrary initial data. One easily sees that the second property of (2.4) is not destroyed; i.e., $\mathbf{V}_{\text {N. s. }} \rightarrow 1 \delta(\mathbf{x})$ as $t \rightarrow 0$ so (3.8) assumes the correct initial data at $t=0$.

4. Evaluation of the fundamental matrix for two dimensions. We consider the twodimensional case of (3.7):

$$
\mathbf{V}_{\mathrm{N} . \mathrm{s} .}^{2}=\sum_{\mu=1}^{4} \frac{1}{(2 \pi)^{2}} \int_{-\infty}^{\infty} \exp \left(-\imath \mathbf{k} \cdot \mathbf{x}-\lambda^{\mu} t\right) \frac{\mathbf{w}^{\mu} \mathbf{w}^{\mu}}{\mathbf{w}^{\mu} \cdot \mathbf{w}^{\mu}} d \mathbf{k}=\sum_{\mu=1}^{4} \mathbf{L}_{\mu}
$$

where the eigenvalues and eigenvectors are given by (3.5) and (3.6). We now want to find the inverse transform $\mathrm{L}_{\mu}$ ( $\mu=1$ to 4 ).

Consider

$$
\mathrm{L}_{1}=\frac{1}{(2 \pi)^{2}} \int_{-\infty}^{\infty} \exp \left(-i \mathbf{k} \cdot \mathbf{z}-\nu k^{2} t\right) d \mathbf{k} \frac{\mathbf{w}^{1} \mathbf{w}^{1}}{\gamma} .
$$

This can easily be integrated to obtain

$$
\mathrm{L}_{1}=\frac{\exp \left(-R^{2} / 4 \nu t\right)}{4 \pi \nu t \gamma} \mathbf{w}^{2} \mathbf{w}^{1}
$$

where $R^{2}=x_{1}^{2}+x_{2}^{2}$.

Next we consider

$$
\mathrm{L}_{2}=-\frac{1}{(2 \pi)^{2}} \int_{-\infty}^{\infty} \exp \left(-i \mathbf{k} \cdot \mathbf{x}-\eta k^{2} t\right) \frac{\mathbf{w}^{2} \mathbf{w}^{2}}{k^{2}} d \mathbf{k}
$$

where $\boldsymbol{w}^{2}=i\left[0, k_{2},-k_{1}, 0\right]$. We observe that all integrals in (4.3) can be obtained from

$$
J_{i j}=\frac{1}{(2 \pi)^{2}} \int_{-\infty}^{\infty} \exp \left(-i \mathbf{k} \cdot \mathbf{x}-\eta k^{2} t\right) \frac{k_{i} k_{i}}{k^{2}} d \mathbf{k} \quad(i, j=1,2) .
$$

A simple invariance argument shows that

$$
J_{i j}(\mathbf{x}, t)=\frac{\delta_{i j}}{2} F^{(1)}(R, t)+\left(\frac{x_{i} x_{i}}{R^{2}}-\frac{\delta_{i j}}{2}\right) F^{(2)}(R, t),
$$

and straightforward calculations show that 


$$
\begin{aligned}
& F^{(1)}(R, t)=\left[\exp \left(-R^{2} / 4 \eta t\right)\right] / 4 \pi \eta t \\
& F^{(2)}(R, t)=-\frac{1}{\pi R^{2}}\left[1-\left(1+\frac{R^{2}}{4 \eta t}\right) \exp \left(-\frac{R^{2}}{4 \eta t}\right)\right]
\end{aligned}
$$

Hence

$$
\mathrm{L}_{2}=\left[\begin{array}{cccc}
0 & 0 & 0 & 0 \\
0 & J_{22} & -J_{12} & 0 \\
0 & -J_{21} & J_{11} & 0 \\
0 & 0 & 0 & 0
\end{array}\right]
$$

To evaluate the remaining two entries of (4.1), note that

$\mathrm{L}_{3}+\mathrm{L}_{4}=2 \operatorname{Re} \mathrm{L}_{3}=2 \operatorname{Re~L}_{4}$

$=-\frac{\operatorname{Re}}{(2 \pi)^{2}} \int_{-\infty}^{\infty} \exp \left(-i \mathbf{k} \cdot \mathbf{x}-i \sqrt{\gamma k t}-\sigma k^{2} t\right) \frac{1}{\gamma k^{2}}\left[\begin{array}{ccc}-k^{2} & \sqrt{\gamma k \mathbf{k}} & -\chi \mathbf{k}^{2} \\ \sqrt{\gamma} k \mathbf{k} & -\gamma \mathbf{k k} & \sqrt{\gamma \chi} k \mathbf{k} \\ -\chi k^{2} & \sqrt{\gamma} \chi k \mathbf{k} & -\chi^{2} k^{2}\end{array}\right] d \mathbf{k}$

The integrals involved in (4.8) are not straightforward. The following types of integrals (not unrelated) enter in the calculation:

$$
\begin{aligned}
M & =\frac{1}{(2 \pi)^{2}} \operatorname{Re} \int_{-\infty}^{\infty} \exp \left(-i \mathbf{k} \cdot \mathbf{x}-i \sqrt{\gamma k} t-\sigma k^{2} t\right) d \mathbf{k} \\
M_{\mathbf{i}} & =\frac{1}{(2 \pi)^{2}} \operatorname{Re} \int_{-\infty}^{\infty} \exp \left(-\imath \mathbf{k} \cdot \mathbf{x}-i \sqrt{\gamma k} t-\sigma k^{2} t\right) \frac{k_{i}}{k} d \mathbf{k} \quad i=1,2 \\
M_{i j} & =\frac{1}{(2 \pi)^{2}} \operatorname{Re} \int_{-\infty}^{\infty} \exp \left(-\imath \mathbf{k} \cdot \mathbf{x}-i \sqrt{\gamma k} t-\sigma k^{2} t\right) \frac{k_{i} k_{i}}{k^{2}} d \mathbf{k} \quad i, j=1,2 .
\end{aligned}
$$

Consistent with the error estimate in (3.7) a second asymptotic analysis may be performed. This is carried out in the appendix and all the entries of (4.9) are evaluated in terms of known functions.

We can now write down the fundamental matrix for the two-dimensional dissipative problem:

$$
\begin{aligned}
\mathrm{V}_{\mathrm{N} . \mathrm{s} .}^{2}= & \frac{\exp \left(-R^{2} / 4 \pi \nu t\right)}{4 \pi \nu t} \frac{\mathrm{w}^{1} \mathbf{w}^{1}}{\gamma} \\
+ & {\left[\begin{array}{cccc}
0 & 0 & 0 & 0 \\
0 & J_{22} & -J_{12} & 0 \\
0 & -J_{21} & J_{11} & 0 \\
0 & 0 & 0 & 0
\end{array}\right] } \\
& +\left[\begin{array}{ccc}
R & \sqrt{\gamma} \times & \chi R \\
\sqrt{\gamma} \times & \gamma \frac{\bar{x}}{R} & \sqrt{\gamma} \chi \mathbf{x} \\
\chi R & \sqrt{\gamma} \chi \mathrm{x} & \chi^{2} R
\end{array}\right] \frac{b(R, t)}{R \gamma}+O^{*}\left(t^{-3 / 2}\right)
\end{aligned}
$$

where $J_{i i}$ is defined by (4.4), (4.5) and (4.6) and $b(R, t)$ is defined by (A8). 
Inviscid limit. We now want to inquire into the inviscid limit of the solution (4.10). First we express the solution of the linearized Euler equations (i.e., setting $\nu, \eta, \sigma=0$ in (4.1)) as

$$
\begin{aligned}
\mathbf{V}_{E}^{2}=\frac{\mathbf{W}^{2} \mathbf{w}^{1}}{\gamma} \delta(\mathbf{x}) & +\left[\begin{array}{cccc}
0 & 0 & 0 & 0 \\
0 & G_{22} & -G_{12} & 0 \\
0 & -G_{21} & G_{11} & 0 \\
0 & 0 & 0 & 0
\end{array}\right] \\
& -\left[\begin{array}{ccc}
0 & \mathbf{x} & 0 \\
\mathbf{x} & 0 & \chi \mathbf{x} \\
0 & \chi \mathbf{x} & 0
\end{array}\right] \frac{H(\sqrt{\gamma} t-R)}{2 \pi \sqrt{\gamma}\left(\gamma t^{2}-R^{2}\right)^{3 / 2}}-\frac{1}{\gamma}\left[\begin{array}{ccc}
W^{(1)} & 0 & \chi W^{(1)} \\
0 & \mathrm{~W} & 0 \\
\chi W^{(1)} & 0 & \chi^{2} W^{(1)}
\end{array}\right]
\end{aligned}
$$

where

$$
\begin{aligned}
G_{i j}(\mathbf{x} t) & =-x_{i} x_{i} / \pi R^{4}+\delta_{i i} /\left(2 \pi R^{2}\right)+\delta(\mathbf{x}) \delta_{i j} / 2 \quad i=1,2, \\
W_{i j}(\mathbf{x}, t) & =\delta_{i j} W^{(1)}(R, t) / 2+\left(x_{i} x_{i} / R^{2}-\delta_{i i} / 2\right) W^{(2)}(R, t) \quad i, j=1,2 \\
W^{(1)} & =\frac{\sqrt{\gamma} t H(\sqrt{\gamma} t-R)}{2 \pi\left(\gamma t^{2}-R^{2}\right)^{3 / 2}} \\
W^{(2)} & =\frac{1}{\pi R^{2}}, \quad R-\sqrt{\gamma} t>0 \\
& =\frac{\left(\sqrt{\gamma} t+2\left(\gamma t^{2}-R^{2}\right)^{1 / 2}\right) R^{2}}{2 \pi\left(\gamma t^{2}-R^{2}\right)^{3 / 2}\left(\sqrt{\gamma} t+\left(\gamma t^{2}-R^{2}\right)^{1 / 2}\right)^{2}}, \quad \sqrt{\gamma} t-R>0 .
\end{aligned}
$$

$\left(H(x)\right.$ and $\delta_{i ;}$ represent the Heaviside function and the Kronecker delta respectively.)

The inviscid limit may be shown to be equivalent in our case to taking $\nu, \eta, \sigma \rightarrow 0$. Imposing this limit, we find

$$
\begin{aligned}
\lim _{\sigma, x, \eta \rightarrow 0} \mathbf{V}_{\mathrm{N} . \mathrm{s} .}^{2}=\frac{\mathbf{w}^{1} \mathbf{w}^{1}}{\gamma} \delta(\mathbf{x}) & +\left[\begin{array}{cccc}
0 & 0 & 0 & 0 \\
0 & G_{22} & -G_{12} & 0 \\
0 & -G_{21} & G_{11} & 0 \\
0 & 0 & 0 & 0
\end{array}\right] \\
& -\left[\begin{array}{ccc}
R & \sqrt{\gamma} \mathrm{x} & \chi R \\
\sqrt{\gamma} \mathrm{x} & \gamma \frac{\mathrm{x}}{R} & \sqrt{\gamma} \chi \mathrm{x} \\
\chi R & \sqrt{\gamma} \chi \mathrm{x} & \chi^{2} R
\end{array}\right] \frac{b^{(1)}(R, t)}{R \gamma}+0^{*}\left(t^{-3 / 2}\right)
\end{aligned}
$$

where $G_{i j}$ is defined by (4.12) and

$$
b^{(1)}(R, t)=\frac{H(\sqrt{\gamma} t-R)}{4 \sqrt{2} \pi \sqrt{R}(\sqrt{\gamma} t-R)^{3 / 2}} .
$$


From (4.11) and (4.14), we observe that the dissipative solution (4.10) does not fully coincide with the nondissipative solution (4.11), the discrepancy being in the region behind the Mach cone. However, the nondissipative solution clearly contains terms of higher order than the error estimate in (4.14). If we consider the region $\sqrt{\gamma} t-R=$ $O\left(t^{1 / 2}\right)$, a simple calculation shows that (4.11) and (4.14) agree there. Therefore in a region of thickness $O\left(t^{1 / 2}\right)$ behind the Mach cone the inviscid limit of (4.10) and the inviscid solution (4.14) agree. If we were to consider the dissipative solution written in terms of Bessel functions, then the nondissipative solution would include the inviscid solution.

It should be noted that the limit in (4.14) is not uniform in time $t$ since no matter how small the dissipation may be, the solution is not inviscid for large time.

5. Evaluation of the fundamental matrix for three dimensions. Setting $n=3$ in (3.7) we obtain

$$
\mathrm{V}_{\mathrm{N} . \mathrm{s} .}^{3}=\sum_{\mu=1}^{5} \frac{1}{(2 \pi)^{3}} \int_{-\infty}^{\infty} \exp \left(-\imath \mathbf{k} \cdot \mathbf{z}-\lambda^{\mu} t\right) \frac{\mathrm{w}^{\mu} \mathrm{w}^{\mu}}{\mathbf{w}^{\mu} \cdot \mathrm{w}^{\mu}} d \mathbf{k}=\sum_{\mu=1}^{5} \mathrm{I}_{\mu} .
$$

We now want to calculate the inverse transforms $I_{\mu}$ for $\mu=1$ to 5 where the eigenvectors and eigenvalues are given by (3.4). The calculation of $I_{1}$ is direct and leads to

$$
I_{1}=\frac{\exp \left(-r^{2} / 4 \nu t\right)}{(4 \pi \nu t)^{3 / 2}} \frac{w^{1} w^{1}}{\gamma}
$$

where $r^{2}=x_{1}^{2}+x_{2}^{2}+x_{3}^{2}$.

Noting that

$\mathbf{I}_{2}+\mathbf{I}_{3}=-\frac{1}{(2 \pi)^{3}} \int_{-\infty}^{\infty} \exp \left(-\imath \mathbf{k} \cdot \mathbf{x}-\eta k^{2} t\right) \frac{1}{k^{2}}\left[\begin{array}{ccccc}0 & 0 & 0 & 0 & 0 \\ 0 & k_{1}^{2}-k^{2} & k_{1} k_{2} & k_{1} k_{3} & 0 \\ 0 & k_{1} k_{2} & k_{2}^{2}-k^{2} & k_{2} k_{3} & 0 \\ 0 & k_{1} k_{3} & k_{2} k_{3} & k_{3}^{2}-k^{2} & 0 \\ 0 & 0 & 0 & 0 & 0\end{array}\right] d \mathbf{k}$

we see that all integrals above may be obtained from

$$
H_{i j}=\frac{1}{(2 \pi)^{3}} \int_{-\infty}^{\infty} \exp \left(-\imath \mathbf{k} \cdot \mathbf{x}-\eta k^{2} t\right) \frac{k_{i} k_{i}}{k^{2}} d \mathbf{k} \quad i, j=1,2,3 .
$$

A simple invariance argument shows that

$$
H_{i j}(\mathbf{x}, t)=\frac{\delta_{i i}}{3} H^{(1)}(r, t)+\left(\frac{x_{i} x_{i}}{r^{2}}-\frac{\delta_{i j}}{3}\right) H^{(2)}(r, t),
$$

and straightforward calculations show that

$$
\begin{gathered}
H^{(1)}(r, t)=\left[\exp \left(-r^{2} / 4 \eta t\right)\right] /(4 \pi \eta t)^{3 / 2} \\
H^{(2)}(r, t)=\frac{\exp \left(-r^{2} / 4 \eta t\right)}{(4 \pi \eta t)^{3 / 2}}-\frac{3}{4 \pi r^{3}} \operatorname{erf}\left(\frac{r}{2(\sqrt{\eta t}}\right)+\frac{3}{2 \pi r^{2}} \frac{\exp \left(-r^{2} / 4 \eta t\right)}{\sqrt{4 \pi \eta t}} .
\end{gathered}
$$




\section{Hence}

$$
\mathrm{I}_{2}+\mathrm{I}_{3}=-\left[\begin{array}{ccccc}
0 & 0 & 0 & 0 & 0 \\
0 & H_{11}-H^{(1)} & H_{12} & H_{13} & 0 \\
0 & H_{12} & H_{22}-H^{(1)} & H_{23} & 0 \\
0 & H_{13} & H_{23} & H_{33}-H^{(1)} & 0 \\
0 & 0 & 0 & 0 & 0
\end{array}\right]
$$

To evaluate the remaining two entries of (5.1), note that

$\mathrm{I}_{4}+\mathrm{I}_{6}=2 \operatorname{Re~I}_{4}=2 \operatorname{Re} I_{5}$

$=-\frac{\operatorname{Re}}{(2 \pi)^{3}} \int_{-\infty}^{\infty} \frac{\exp \left(-i \mathbf{k} \cdot \mathbf{x}-i \sqrt{\gamma} k t-\sigma k^{2} t\right)}{\gamma k^{2}}\left[\begin{array}{ccc}-k^{2} & \sqrt{\gamma} k \mathbf{k} & -\chi k^{2} \\ \sqrt{\gamma} k \mathbf{k} & -\gamma \mathbf{k k} & \sqrt{\gamma} \chi k \mathbf{k} \\ -\chi k^{2} & \sqrt{\gamma} \chi k \mathbf{k} & -\chi^{2} k^{2}\end{array}\right] d \mathbf{k}$.

Again we see that the following integrals (not unrelated) enter in the calculation:

$$
\begin{aligned}
N & =\frac{\operatorname{Re}}{(2 \pi)^{3}} \int_{-\infty}^{\infty} \exp \left(-i \mathbf{k} \cdot \mathbf{x}-i \sqrt{\gamma} k t-\sigma k^{2} t\right) d \mathbf{k} \\
N_{i} & =\frac{\operatorname{Re}}{(2 \pi)^{3}} \int_{-\infty}^{\infty} \exp \left(-i \mathbf{k} \cdot \mathbf{x}-i \sqrt{\gamma} k t-\sigma k^{2} t\right) \frac{k_{i}}{k} d \mathbf{k} \\
N_{i j} & =\frac{\operatorname{Re}}{(2 \pi)^{3}} \int_{-\infty}^{\infty} \exp \left(-i \mathbf{k} \cdot \mathbf{x}-i \sqrt{\gamma} k t-\sigma k^{2} t\right) \frac{k_{i} k_{i}}{k^{2}} d \mathbf{k} .
\end{aligned}
$$

Before proceeding further with the calculations, we pause to mention that a finer analysis of the error terms shows that instead of (3.7) we can write

$$
\mathrm{V}^{3}=\mathrm{V}_{\mathrm{N} . \mathrm{s} .}+\sigma O\left(t^{-3 / 2}\right) \text {. }
$$

It also shows that this error term vanishes with vanishing viscosity. It is for this reason that we indicate the coefficient $\sigma$ in the above.

A simple invariance argument states that

$$
N_{i j}(\mathrm{x}, t)=\left(\delta_{i i} / 3\right) N(r, t)+\left(x_{i} x_{j} / r^{2}-\delta_{i j} / 3\right) D^{(1)}(r, t) \quad i, j=1,2,3
$$
and

$$
N_{i}=\left(x_{i} / r\right) D^{(2)}(r, t) \quad i=1,2,3 .
$$

Using straightforward calculations (Gradstehyn and Ryzhik [3]), it can easily be shown that

$$
\begin{aligned}
N(r, t) & =\frac{(r-\sqrt{\gamma} t)}{2 r(4 \pi \sigma t)^{3 / 2}} \exp \left(\frac{-(r-\sqrt{\gamma} t)^{2}}{4 \sigma t}\right)+O^{*}\left(t^{-11 / 4}\right) \\
& =d(r, t)+O^{*}\left(t^{-11 / 4}\right), \\
D^{(1)}(r, t) & =\frac{(r-\sqrt{\gamma} t)}{2 r(4 \pi \sigma t)^{3 / 2}} \exp \left(\frac{-(r-\sqrt{\gamma} t)^{2}}{4 \sigma t}\right) \\
& \quad+\frac{3}{4 \pi r^{2}(4 \pi \sigma t)^{1 / 2}} \exp \left(\frac{-(r-\sqrt{\gamma} t)^{2}}{4 \sigma t}\right)+O^{*}\left(t^{-11 / 4}\right)
\end{aligned}
$$

and

$$
\begin{aligned}
D^{(2)}(r, t)=\frac{(r-\sqrt{\gamma} t)}{2 r(4 \pi \sigma t)^{3 / 2}} \exp \left(\frac{-(r-\sqrt{\gamma} t)^{2}}{4 \sigma t}\right) & \\
& -\frac{1}{4 \pi r^{2}(4 \pi \sigma t)^{1 / 2}} \exp \left(\frac{-(r-\sqrt{\gamma} t)^{2}}{4 \sigma t}\right)+O^{*}\left(t^{-11 / \eta}\right) .
\end{aligned}
$$


We can now write down the fundamental matrix for the three-dimensional dissipative problem:

$$
\begin{aligned}
\mathbf{V}_{\mathrm{N} . \mathrm{s} .}^{3}=\frac{\exp \left(-\gamma^{2} / 4 \nu t\right)}{(4 \pi \nu)^{3 / 2}} \frac{\mathrm{w}^{1} \mathrm{w}^{1}}{\gamma}-\left[\begin{array}{ccccc}
0 & 0 & 0 & 0 & 0 \\
0 & H_{11}-H^{(1)} & H_{12} & H_{13} & 0 \\
0 & H_{12} & H_{22}-H^{(1)} & H_{23} & 0 \\
0 & H_{13} & H_{23} & H_{33}-H^{(1)} & 0 \\
0 & 0 & 0 & 0 & 0
\end{array}\right] \\
+ \\
+\left[\begin{array}{lll}
1 & 0 & \chi \\
0 & 0 & 0 \\
\chi & 0 & \chi^{2}
\end{array}\right] \frac{d(r, t)}{\gamma}+\left[\begin{array}{ccc}
0 & 0 & 0 \\
0 & N_{i i} & 0 \\
0 & 0 & 0
\end{array}\right]+\left[\begin{array}{ccc}
0 & \sqrt{\gamma} \mathbf{x} & 0 \\
\sqrt{\gamma} \mathbf{x} & 0 & \sqrt{\gamma} x \mathbf{x} \\
0 & \sqrt{\gamma} x \mathbf{x} & 0
\end{array}\right] \\
+\left(\frac{1}{4 \pi r^{3}(4 \pi \sigma t)^{1 / 2} \gamma} \exp \left(\frac{-(r-\sqrt{\gamma} t)^{2}}{4 \sigma t}\right)\right. \\
\left.+\frac{(r-\sqrt{\gamma} t)}{2 r^{2} \gamma(4 \pi \sigma t)^{3 / 2}} \exp \left(\frac{-(r-\sqrt{\gamma} t)^{2}}{4 \sigma t}\right)\right)+O^{*}\left(t^{-11 / 4}\right) .
\end{aligned}
$$

We note that in (5.12) we carry terms of $O\left(t^{-5 / 2}\right)$. However, as we pointed out above, terms of $O\left(t^{-5 / 2}\right)$ have already been neglected in obtaining $\mathrm{V}_{N . s .}^{3}$. . There is, however, an important distinction between terms carried and those neglected. For in fact the latter can be shown to vanish in the limit of vanishing dissipation, whereas the terms retained in (5.12) do not vanish in this limit.

The solution appears as diffuse waves travelling outwards with a speed $\sqrt{\gamma}$ and pure diffusion modes (entropy and vorticity waves) which remain at the origin. The speed $\sqrt{\gamma}$ is just the adiabatic speed of sound in our normalization.

Inviscid limit. The solution of the linearized Euler equations (i.e., setting $\nu, \eta, \sigma=0$ in (5.1)) can be expressed as

$$
\begin{aligned}
\mathbf{V}_{E}^{3}=\frac{\mathrm{w}^{1} \mathbf{w}^{1}}{\gamma} \delta(\mathrm{x}) & -\left[\begin{array}{ccccc}
0 & 0 & 0 & 0 & 0 \\
0 & P_{11}-\delta(\mathrm{x}) & P_{12} & P_{13} & 0 \\
0 & P_{12} & P_{22}-\delta(\mathrm{x}) & P_{23} & 0 \\
0 & P_{13} & P_{23} & P_{33}-\delta(\mathrm{x}) & 0 \\
0 & 0 & 0 & 0 & 0
\end{array}\right] \\
& -\left[\begin{array}{ccc}
1 & 0 & \chi \\
0 & 0 & 0 \\
\chi & 0 & \chi^{2}
\end{array}\right] \frac{c(r, t)}{\gamma}+\left[\begin{array}{ccc}
0 & 0 & 0 \\
0 & C_{i i} & 0 \\
0 & 0 & 0
\end{array}\right] \\
+ & {\left[\begin{array}{ccc}
0 & \sqrt{\gamma} \mathrm{x} & 0 \\
\sqrt{\gamma} \mathrm{x} & 0 & \sqrt{\gamma} \chi \mathrm{x} \\
0 & \sqrt{\gamma} \chi \mathbf{x} & 0
\end{array}\right]\left(\delta(r-\sqrt{\gamma} t) /\left(4 \pi r^{3} \gamma\right)-c(r, t) / r \gamma\right) }
\end{aligned}
$$


where

$$
\begin{aligned}
c(r, t) & =\delta^{\prime}(r-\sqrt{\gamma} t) /(4 \pi r) \\
P_{i i} & =\frac{\delta_{i j}}{4 \pi r^{3}}-\frac{3 x_{i} x_{j}}{4 \pi r^{5}}+\frac{\delta_{i j}}{3} \delta(\mathbf{x})
\end{aligned}
$$

and

$C_{i j}=-\frac{\delta_{i j}}{3} c(r, t)+\left(\frac{x_{i} x_{i}}{r^{2}}-\frac{\delta_{i j}}{3}\right)\left(-c(r, t)+\frac{3}{4 \pi r^{2}} \delta(r-\sqrt{\gamma} t)-\frac{3}{8 \pi r^{3}} \operatorname{sgn}(r-\sqrt{\gamma} t)\right)$,

(where sgn $(x)$ represents the signum function).

Performing the nondissipative limit (i.e. $\nu, \eta, \sigma \rightarrow 0$ ) on the three-dimensional fundar mental matrix (5.14), we obtain

$$
\begin{aligned}
& \lim _{x, y, \eta \rightarrow 0} \mathrm{~V}_{\mathrm{N} . \mathrm{s} .}^{3}=\frac{\mathrm{w}^{1} \mathbf{w}^{1}}{\gamma} \delta(\mathrm{x})-\left[\begin{array}{ccccc}
0 & 0 & 0 & 0 & 0 \\
0 & P_{11}-\delta(\mathrm{x}) & P_{12} & P_{13} & 0 \\
0 & P_{12} & P_{22}-\delta(\mathrm{x}) & P_{23} & 0 \\
0 & P_{13} & P_{23} & P_{33}-\delta(\mathrm{x}) & 0 \\
0 & 0 & 0 & 0 & 0
\end{array}\right] \\
& +\left[\begin{array}{ccc}
0 & \sqrt{\gamma} \mathbf{x} & 0 \\
\sqrt{\gamma} \mathbf{x} & 0 & \sqrt{\gamma} \chi \mathbf{x} \\
0 & \sqrt{\gamma} \chi \mathbf{x} & 0
\end{array}\right] \frac{\delta(r-\sqrt{\gamma} t)}{4 \pi r^{3} \gamma}-\frac{c(r, t)}{r \gamma} \\
& -\left[\begin{array}{lll}
1 & 0 & \chi \\
0 & 0 & 0 \\
\chi & 0 & \chi^{2}
\end{array}\right] \frac{c(r, t)}{\gamma}+\left[\begin{array}{ccc}
0 & 0 & 0 \\
0 & C_{i j}^{(1)} & 0 \\
0 & 0 & 0
\end{array}\right]+O^{*}\left(t^{-11 / 4}\right)
\end{aligned}
$$

where

$C_{i j}^{(1)}=-\frac{\delta_{i j}}{3} c(r, t)+\left(\frac{x_{i} x_{i}}{r^{2}}-\frac{\delta_{i j}}{3}\right)\left(-c(r, t)+\frac{3}{4 \pi r^{2}} \delta(r-\sqrt{\gamma} t)\right) \quad(i, j=1,2,3)$

and $P_{i j}$ and $c(r, t)$ are defined by (5.14). Again we observe that the inviscid limit (5.15) does not fully coincide with the inviscid solution (5.13). However, this discrepancy is due entirely to the dropping of higher-order terms in our fundamental matrix (5.12). It should again be noted that the limit in (5.15) is not uniform in time $t$ since not matter how small the dissipation may be, the solution is not inviscid for large time.

The reason the inviscid limit includes the inviscid solution in three dimensions and not two dimensions is simply due to the difference in properties of the wave equation in even and odd dimensions.

6. Period of final decay. Consider the solution to the initial value problem for compact support initial data of the form

$$
\nabla_{0}=\nabla_{0}(x)=0 \text { for }|x|>\mathscr{L} / L
$$

where $\mathscr{L}$ is a measure of the extent of the initial disturbance in dimensional units. Taking 
the Fourier transform of (6.1), we obtain

$$
\mathrm{v}_{0}(\mathbf{k})=\int_{|\mathbf{x}|<\mathcal{L} / L} e^{i \mathbf{k} \cdot \mathbf{x}} \nabla_{0}(\mathbf{x}) d \mathbf{x} .
$$

In a straightforward expansion $\nabla_{0}(\mathbf{k})=\mathrm{f}+O(i k \mathcal{L} / L)$ where $\mathrm{f}=\int_{-\infty}^{\infty} \nabla_{0}(\mathbf{x}) d \mathbf{x}$ is identified with the total mass, momentum and energy of the initial data. Applying the same procedure used in finding the fundamental matrix, we now have for the solution to the initial value problem (6.1)

$$
\nabla^{n}=\mathrm{V}_{\mathrm{N} . \mathrm{s.}}^{n} \cdot \mathbf{f}+\frac{\mathfrak{L}}{L} O^{*}\left(t^{-8 n / 4}\right) \quad(n=2,3) .
$$

From (4.10) and (5.14), we therefore have $\nabla^{n} \sim \nabla_{f}^{n}=V_{N . s .}^{n} \cdot f(n=2,3)$. For dimensional time, $\tau, \tau \gg(\mathscr{L} / L)^{3}\left(\mathscr{L} / a_{0}\right)$. The right-hand side is the product of the time it takes a sound wave to traverse the initial disturbance with the inverse of a Knudsen number based on the extent of initial disturbance. $\tau$ is referred to the period of final decay. In summary, the period of final decay is described by the fundamental solution $\nabla_{f}^{n}(n=2,3)$ associated with the total mass, momentum, and energy addition of the initial disturbance. Compact support initial data was chosen for convenience. However, by more careful estimates the same results hold, for example, for initial data which is of exponential type at infinity. It should be emphasized that the asymptotic solution using the convolution is less severe, i.e. we can write

$$
\nabla^{n}=\mathrm{V}_{N . S .}^{n} * \nabla_{0}+O^{*}\left(t^{-3 n / 4}\right) \quad(n=2,3)
$$

with a modulus in the error estimate of $O(1)$ instead of $\mathscr{L} / L$.

Appendix: second asymptotic analysis. The asymptotic approximation of integrals of the type (4.9) has been given elsewhere [9]. Due to certain variations and for completeness we outline such a discussion here. To evaluate these integrals we first introduce the position vector $\mathbf{x}$ as the reference axis of integration $\mathbf{x}=R \mathrm{e}_{1}$ in terms of which we write $\mathbf{k}=k \cos \theta \mathbf{e}_{1}+k \sin \theta \mathbf{e}_{2}$. Then let us first consider $M$ in (4.9). We have directly

$$
M=\frac{1}{2 \pi} \operatorname{Re} \int_{0}^{\infty} k J_{0}(k R) \exp \left(-i \sqrt{ } \gamma k t-\sigma k^{2} t\right) d k
$$

where $J_{0}$ is the Bessel function of zero order. We first consider the region $R=o(t)$ where as usual $t \rightarrow \infty$. This analysis is straightforward and we find $M \sim 1 /\left(2 \pi \gamma t^{2}\right)$, which is an already neglected order. We therefore restrict attention to $R \geq O(t)$. Let $p>0$ and consider

$$
Q=\frac{1}{2 \pi} \operatorname{Re} \int_{0}^{o(t-D)} k J_{0}(k R) \exp \left(-i \sqrt{\gamma} k t-\sigma k^{2} t\right) d k
$$

then

$$
O(Q) \leq \int_{0}^{t-D} k J_{0}(k R) d k
$$

The integral may be directly integrated and we find

$$
O(Q) \leq J_{1}\left(R t^{-D}\right) / t^{p} R \text {. }
$$


Take $p<1$; in view of $R \geq O(t)$, we can use the asymptotic approximations for the Bessel function $J_{1}$ and find $O(Q) \leq\left(1 /\left(R^{3 / 2} t^{p / 2}\right)\right)$, which is clearly of neglected order.

Next we consider

$$
M-Q=\frac{1}{2 \pi} \operatorname{Re} \int_{O(t-D)}^{\infty} J_{0}(k R) k \exp \left(-i \sqrt{\gamma} k t-\sigma k^{2} t\right) d k .
$$

Since the argument of the Bessel function is large, we may write

$$
\left.J_{0}(k R)=(2 / \pi k R)^{1 / 2} \cos (k R-(\pi / 4))+O\left(1 /(k R)^{3 / 2}\right)\right) .
$$

Consider

$$
A=\frac{1}{2 \pi} \operatorname{Re} \int_{O(t-D)}^{\infty}\left[J_{0}(k R)-\left(\frac{2}{\pi k R}\right)^{1 / 2} \cos \left(k R-\frac{\pi}{4}\right)\right] \exp \left(-i \sqrt{\gamma} k t-\sigma k^{2} t\right) k d k ;
$$

then, using straightforward estimates, we find $O(A) \leq 1 / R t^{3(1-p) / 2}$. It only remains for us to consider

$$
A^{(1)}=\frac{1}{2 \pi} \operatorname{Re} \int_{0}^{o(t-D)} k\left(\frac{2}{\pi k R}\right)^{1 / 2} \cos \left(k R-\frac{\pi}{4}\right) \exp \left(-i \sqrt{\gamma} k t-\sigma k^{2} t\right) d k,
$$

for which we easily find $O\left(A^{(1)}\right)<1 /\left(R^{1 / 2} t^{3 p / 2}\right)$. Therefore taking, for example, $p=\frac{2}{3}$ we have demonstrated that

$$
M=\frac{\mathrm{Re}}{\left(2 \pi^{3} R\right)^{1 / 2}} \int_{0}^{\infty} k^{1 / 2} \cos \left(k R-\frac{\pi}{4}\right) \exp \left(-i \sqrt{ } \gamma k t-\sigma k^{2} t\right) d k+O\left(t^{-3 / 2}\right) .
$$

Next consider $M_{\text {i }}$ of (4.9). A simple invariance argument states

$$
M_{i}=\left(x_{i} / R\right) B(R, t) \quad i=1,2,
$$

and we easily show

$$
B(R, t)=-\frac{\mathrm{Re}}{2 \pi} i \int_{0}^{\infty} \exp \left(-i \sqrt{\gamma} k t-\sigma k^{2} t\right) J_{1}(k R) k d k .
$$

The same arguments and estimates used in obtaining (A1) may be applied to (A3). It suffices to say that we can replace $J_{1}(k R)$ by its asymptotic approximation and write $B(R, t)=-\frac{\mathrm{Re}}{\left(2 \pi^{3} R\right)^{1 / 2}} i \int_{0}^{\infty} k^{1 / 2} \cos \left(k R-\frac{\pi}{4}\right) \exp \left(-i \sqrt{\gamma} k t-\sigma k^{2} t\right) d k+O\left(t^{-3 / 2}\right)$.

Finally, considering $M_{i j}$ of (4.9), we can write

$$
M_{i i}=\frac{\delta_{i i}}{2} M(R, t)+\left(\frac{x_{i} x_{i}}{R^{2}}-\frac{\delta_{i j}}{2}\right) B^{(1)}(R, t) \quad i, j=1,2,
$$

where $M(R, t)$ is given by (4.9) and

$$
B^{(1)}(R, t)=-\frac{\operatorname{Re}}{2 \pi} \int_{0}^{\infty} k e^{-i \sqrt{\gamma} k t-\sigma k^{2} t} J_{2}(k R) d k ;
$$

using previous arguments, (A6) becomes $B^{(1)}(R, t)$

$$
=\frac{\operatorname{Re}}{\left(2 \pi^{3} R\right)^{1 / 2}} \int_{0}^{\infty} k^{1 / 2} \cos \left(k R-\frac{\pi}{4}\right) \exp \left(-i \sqrt{\gamma} k t-\sigma k^{2} t\right) d k+O\left(t^{-3 / 2}\right) .
$$


The integral occurring in (A7) is the same as that appearing in (A1). Therefore this and the integral in (A4) are the only integrals which need to be evaluated.

Both integrals may be evaluated using standard integral tables. In an obvious notation

$$
B^{(1)}(R, t)=b(R, t)+O\left(t^{-3 / 2}\right)
$$

we find

$$
\begin{aligned}
& b(R, t)=\frac{\Gamma(3 / 4)}{8 R^{1 / 2} \pi^{3 / 2}(\sigma t)^{3 / 4}} M\left(\frac{3}{4}, \frac{1}{2},-\frac{(R-\sqrt{\gamma} t)^{2}}{4 \sigma t}\right) \\
& +\frac{\Gamma(5 / 4)(R-\sqrt{\gamma} t) \exp \left(-(R-\sqrt{\gamma} t)^{2} / 4 \sigma t\right)}{8 R^{1 / 2} \pi^{3 / 2}(\sigma t)^{5 / 4}} M\left(\frac{1}{4}, \frac{3}{2}, \frac{(R-\sqrt{\gamma} t)^{2}}{4 \sigma t}\right),
\end{aligned}
$$

where $M(a, b, z)$ is the confluent hypergeometric function [1]. A similar argument for $B(R, t)$, (A4), shows

$$
B(R, t)=-b(R, t)+O\left(t^{-3 / 2}\right) .
$$

In resume, we have shown $M=b(R, t)+O\left(t^{-3 / 2}\right)$,

$$
\begin{gathered}
M_{i}=-\frac{x_{i}}{R} b(R, t)+O\left(t^{-3 / 2}\right) \quad i=1,2, \\
M_{i i}=\frac{x_{i} x_{i}}{R^{2}} b(R, t)+O\left(t^{-3 / 2}\right) \quad i, j=1,2 .
\end{gathered}
$$

\section{REFERENCES}

[1] M. Abramowitz and I. A. Stegun (editors), Handbook of mathematical functions, with formulas, graphs and mathematical tables, Nat. Bur. Standards Appl. Math. Series, 55, U.S. Government Printing Office, Washington, D.C., 1964

[2] I. M. Gel'fand and G. E. Silov, General functions. Vol. I: Properties and operations, Fizmatgiz, Moscow, 1958; English transl., Academic Press, New York, 1964

[3] I. S. GradsteY̌n, and I. M. Ryžik, Tables of integrals, series and products, Fizmatgiz, Moscow, 1963; English transl., Academic Press, New York, 1965.

[4] W. D. Hayes, Gasdynamic discontinuities, Princeton Aeronautical Paperbacks, Princeton, N. J., 1960

[5] M. J. Lighthill, Viscosity effects in sound waves of finite amplitude, Surveys in Mechanics, Cambridge Univ. Press, Cambridge, 1956, pp. 250-351

[6] J. P. Moran and S. F. Shen, On the formation of weak plane shock waves by impulsive motion of a piston, J. Fluid Mech. 25, 705-718 (1966)

[7] O. S. Ryżov, Influence of viscosity and thermal conductivity on propagation of sound impulses, Prikl. Mat. Mch. 30, 296-302 (1966) = J. Appl. Math. Mech. 30, 362-369 (1966)

[8] L. Sirovich, Initial and boundary value problems in dissipative gas dynamics, Phys. Fluids 10, 24-34 (1967)

[9] L. Sirovich, The asymptotic evaluation of multidimensional integrals, J. Math. Phys. 11, 1365-1374 (1970)

[10] C. H. Su and G. S. Gardner, Derivation of the Korteweg de Vries equation and Burgers equation, J. Math. Phys. 10, 536-539 (1969) 